\title{
Reconfigurable Flow Platform for Automated Reagent Screening and Autonomous Optimization for Bio-inspired Lignans Synthesis
}

Ehu Camille Aka, ${ }^{\ddagger}$ Eric Wimmer, ${ }^{\ddagger}$ Elvina Barré, ${ }^{\ddagger}$ Natarajan Vasudevan, ${ }^{\ddagger}$ Daniel CortésBorda, ${ }^{\ddagger}$ Tchirioua Ekou,${ }^{\dagger}$ Lynda Ekou,${ }^{\dagger}$ Mireia Rodriguez-Zubiri,,${ }^{\ddagger}$ François-Xavier Felpin* $*$

¥ Université de Nantes, UFR des Sciences et des Techniques, CNRS UMR 6230, CEISAM, 2 rue de la Houssinière, 44322 Nantes Cedex 3, France

$\dagger$ Université Nangui Abrogoua, Laboratoire de Thermodynamique et de Physico-Chimie du Milieu, 02 BP 801 Abidjan 02, Côte d'Ivoire

\section{Table of content}

1. Optimization algorithm S2

2. Optimization studies for the synthesis benzoxanthenone (10) S2

3. Photograph of the automated screening platform S4

4. NMR Spectra of compounds $6,10,12,1,13,14,15,16,17,18$ and $3 \quad$ S5-S14

5. References $\quad$ S15 


\section{Optimization algorithm}

The optimization algorithm here used has been described in our previous reports..$^{1-2}$

\section{Optimization studies for the synthesis benzoxanthenone (10)}

TABLE S1. Maximization of the reaction yield of benzoxanthenone (10).

\begin{tabular}{|c|c|c|c|c|c|c|}
\hline Run & $\begin{array}{l}{[\mathrm{Co}]-9 \mathbf{a}} \\
\text { (equiv) }\end{array}$ & $\begin{array}{c}t \text {-BuOOH } \\
\text { - } \mathbf{8 a} \\
\text { (equiv) }\end{array}$ & $\begin{array}{c}\text { Residence } \\
\text { time } \\
(\min )\end{array}$ & $\begin{array}{c}\text { Temperature } \\
\left({ }^{\circ} \mathrm{C}\right)\end{array}$ & $\begin{array}{l}\text { Yield } \\
(\%)\end{array}$ & $\begin{array}{c}\text { Productivity } \\
\text { (mg/h) }\end{array}$ \\
\hline 1 & 0.050 & 1.00 & 5 & 25 & 44 & 1359 \\
\hline 2 & 0.050 & 1.00 & 15 & 25 & 62 & 638 \\
\hline 3 & 0.050 & 1.00 & 5 & 30 & 60 & 1853 \\
\hline 4 & 0.050 & 1.20 & 5 & 25 & 52 & 1557 \\
\hline 5 & 0.070 & 1.00 & 5 & 25 & 52 & 1327 \\
\hline 6 & 0.060 & 1.10 & 10 & 28 & 58 & 799 \\
\hline 7 & 0.058 & 1.00 & 9 & 27 & 59 & 934 \\
\hline 8 & 0.039 & 1.05 & 15 & 30 & 67 & 773 \\
\hline 9 & 0.024 & 1.08 & 19 & 32 & 72 & 792 \\
\hline 10 & 0.042 & 1.00 & 13 & 29 & 65 & 843 \\
\hline 11 & 0.037 & 1.04 & 23 & 27 & 67 & 517 \\
\hline 12 & 0.018 & 1.06 & 26 & 29 & 76 & 671 \\
\hline 13 & 0.010 & 1.07 & 29 & 30 & 86 & 776 \\
\hline 14 & 0.010 & 1.08 & 26 & 33 & 82 & 823 \\
\hline 15 & 0.010 & 1.10 & 30 & 31 & 84 & 727 \\
\hline 16 & 0.010 & 1.09 & 27 & 32 & 92 & 887 \\
\hline \multicolumn{7}{|c|}{ Dimension reduction } \\
\hline 17 & 0.010 & 1.08 & 31 & 29 & 95 & 800 \\
\hline 18 & 0.010 & 1.08 & 33 & 27 & 77 & 609 \\
\hline 19 & 0.010 & 1.06 & 28 & 29 & 81 & 759 \\
\hline 20 & 0.010 & 1.09 & 30 & 31 & 85 & 738 \\
\hline 21 & 0.010 & 1.11 & 29 & 32 & 83 & 741 \\
\hline 22 & 0.010 & 1.08 & 29 & 30 & 89 & 801 \\
\hline 23 & 0.010 & 1.08 & 28 & 30 & 89 & 830 \\
\hline 24 & 0.010 & 1.09 & 28 & 31 & 80 & 744 \\
\hline
\end{tabular}

Stopping criterion reached - No improvement after 5 consecutive simplexes 


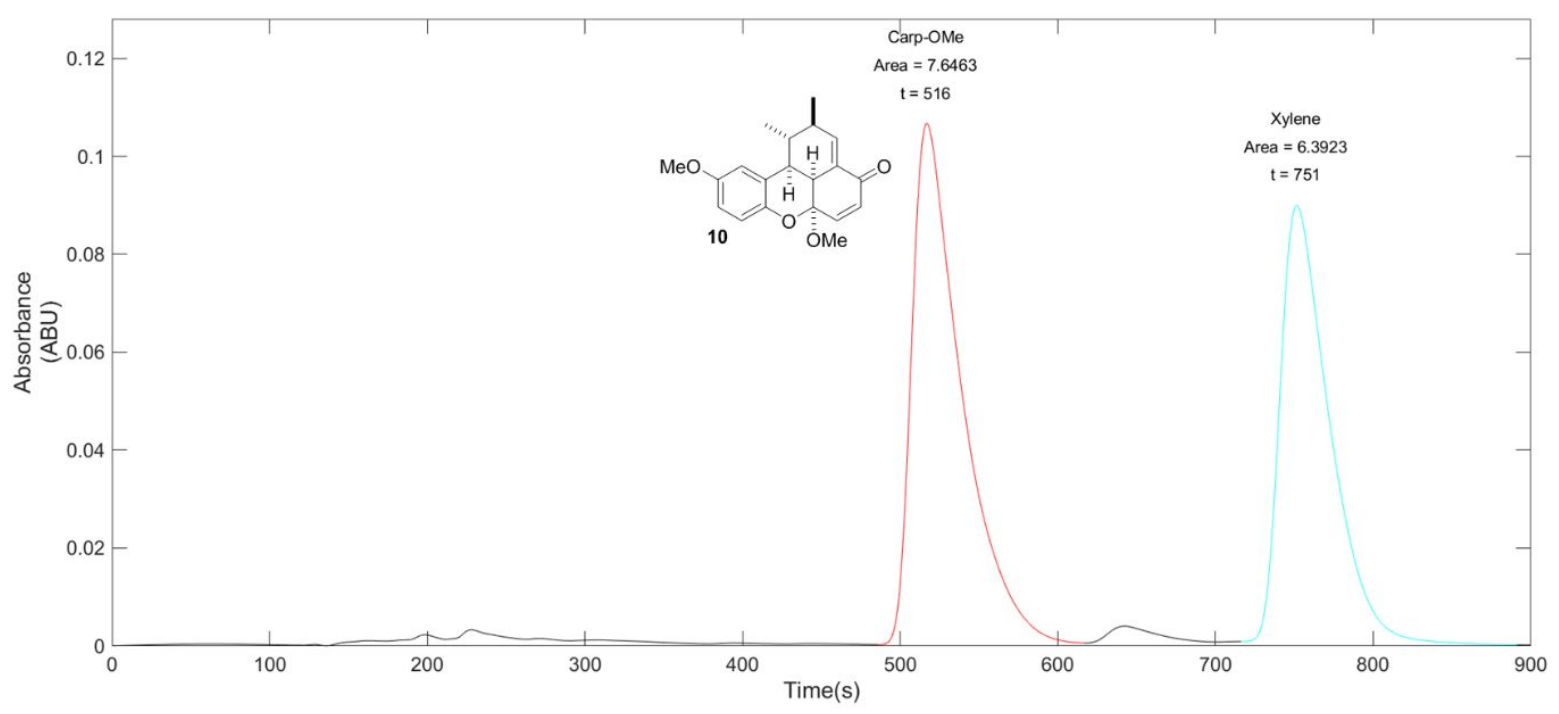

FIGURE S1. HPLC chromatogram of the crude mixture of experiment 16. 


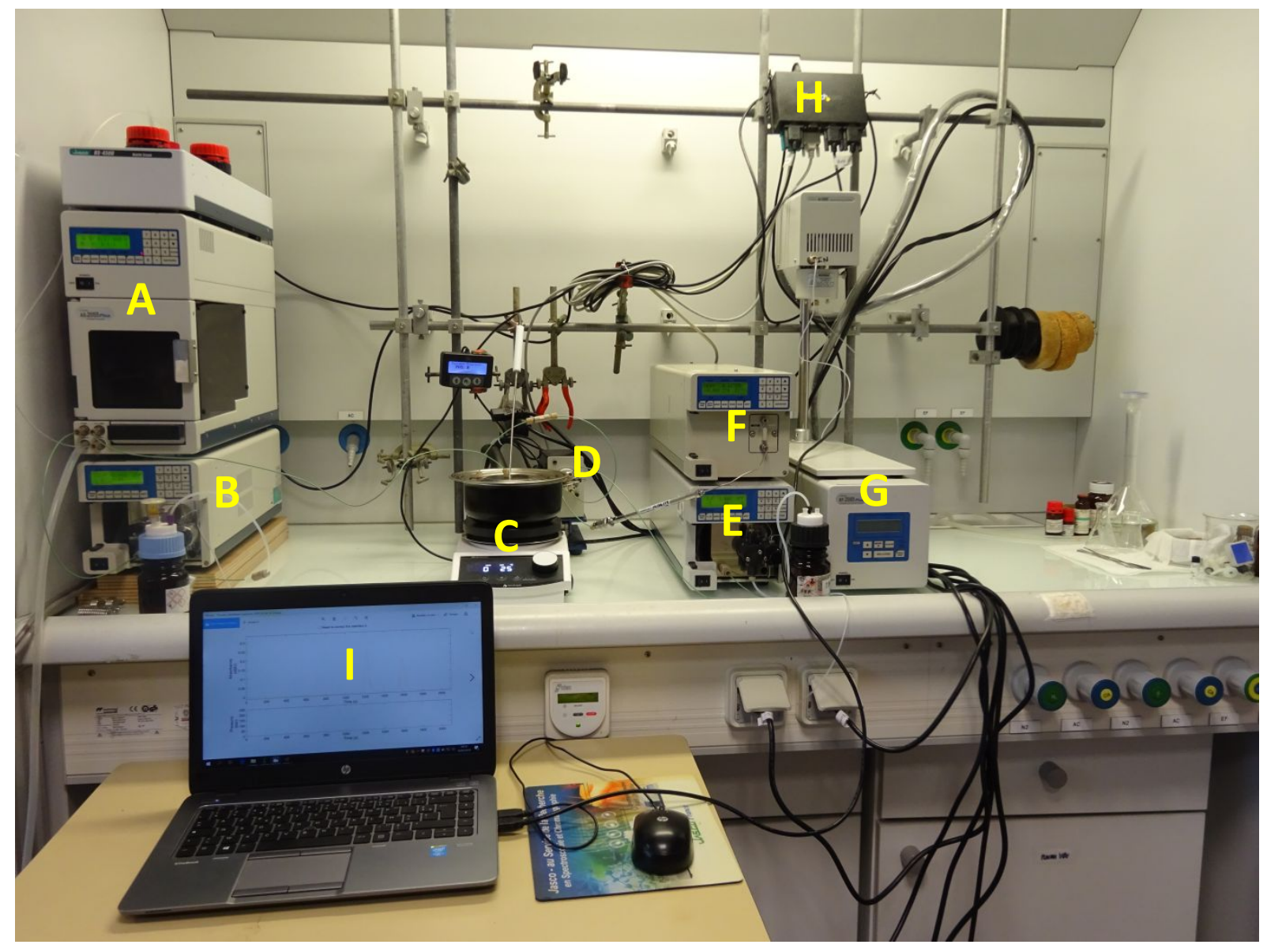

FIGURE S2. Experimental setup for the automated screening. A: liquid handling robot. B: pump. C: Heated reactor. D: Automated 2-way 6-port valve. E: HPLC pump. F: UV detector. G: Optional electronic back pressure regulator. H: USB to RS-232 port serial hub. I: Computer connected to the serial hub. 


\section{NMR spectra}

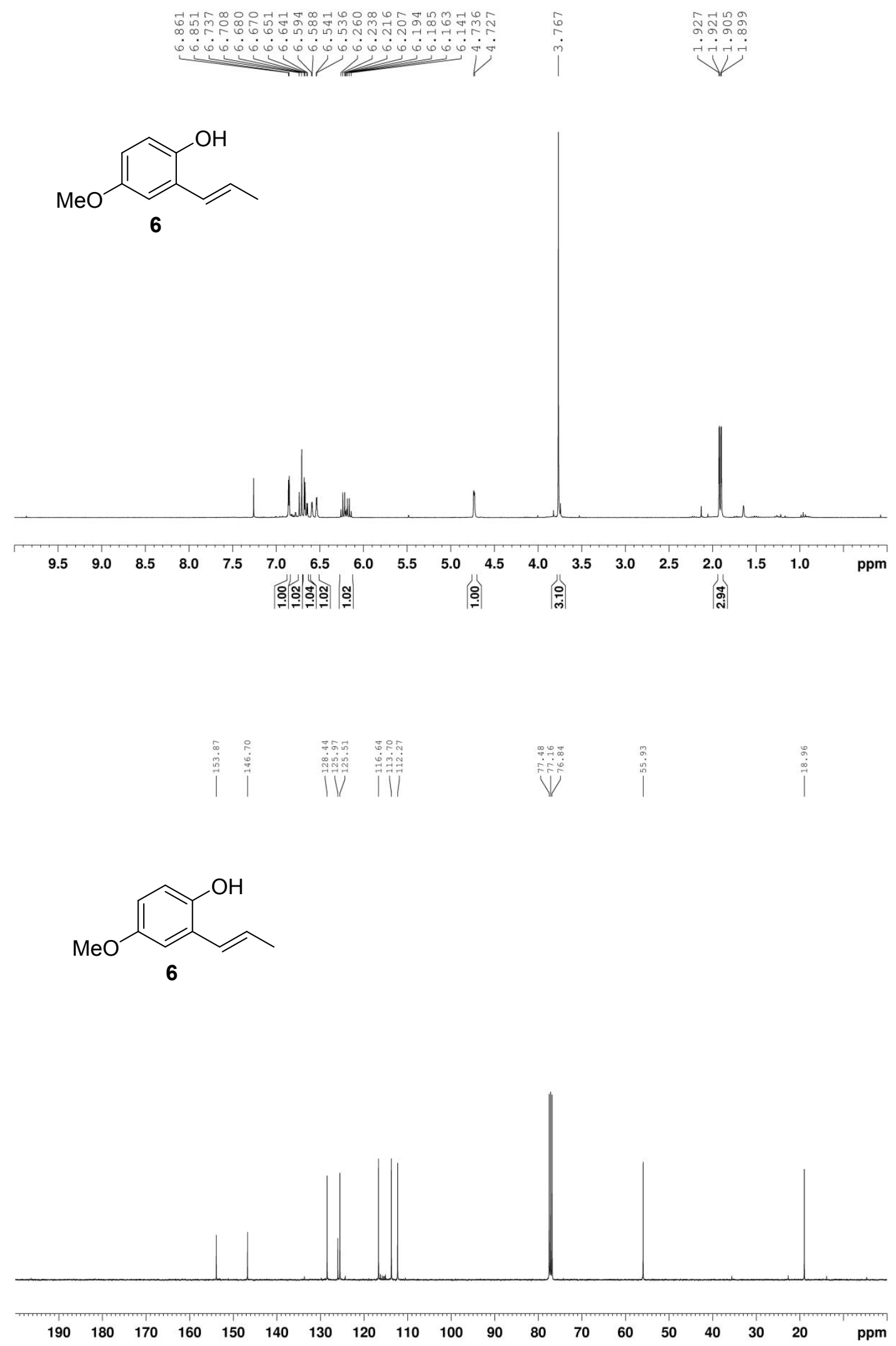




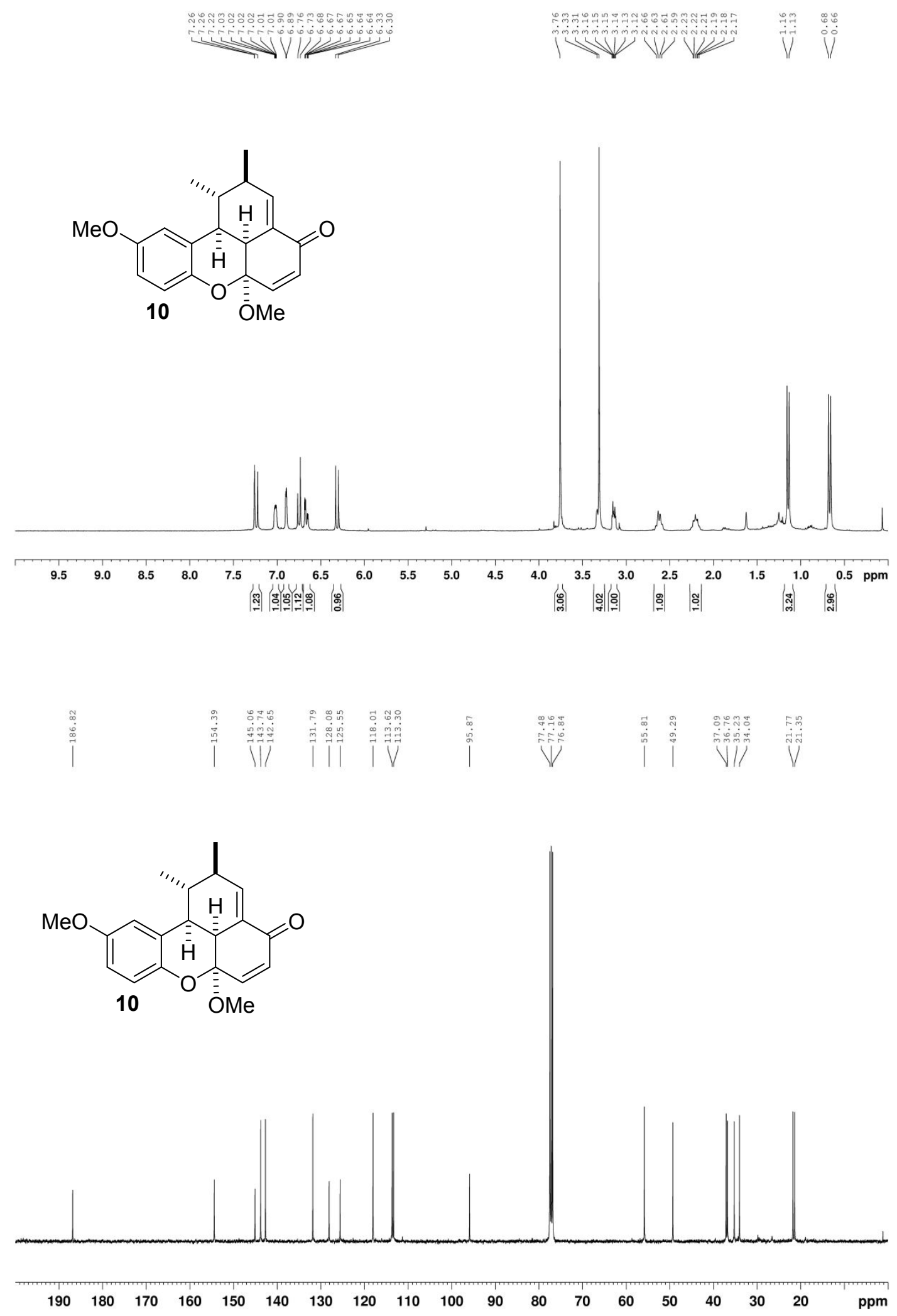




\section{|vi|| ||}

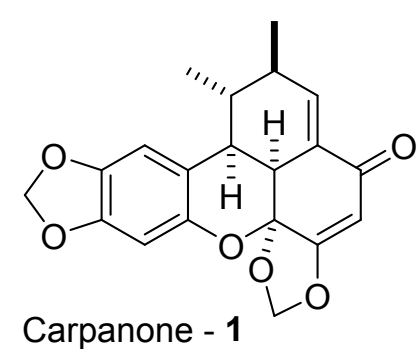
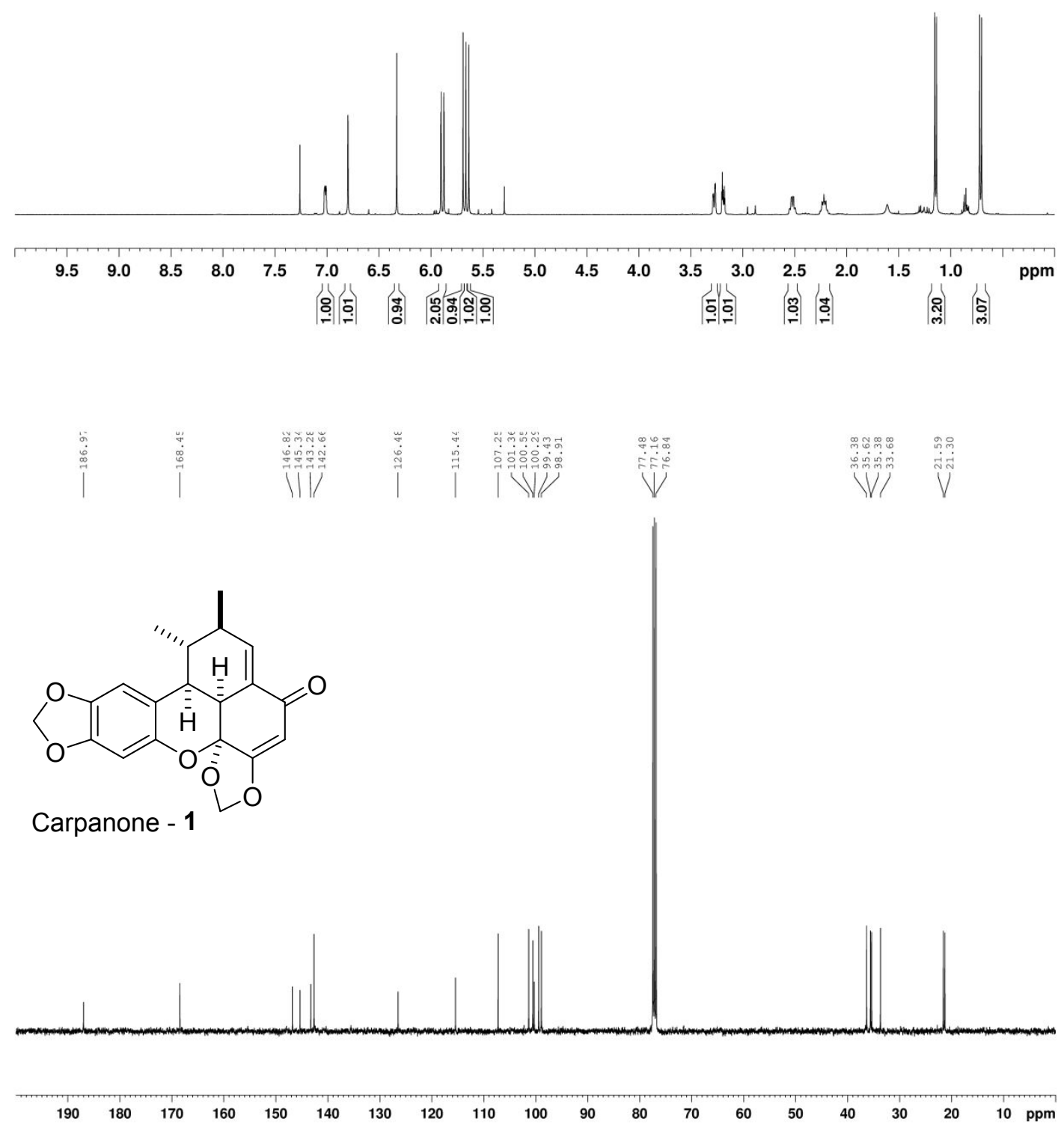


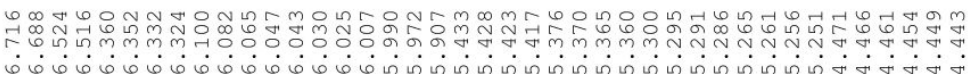
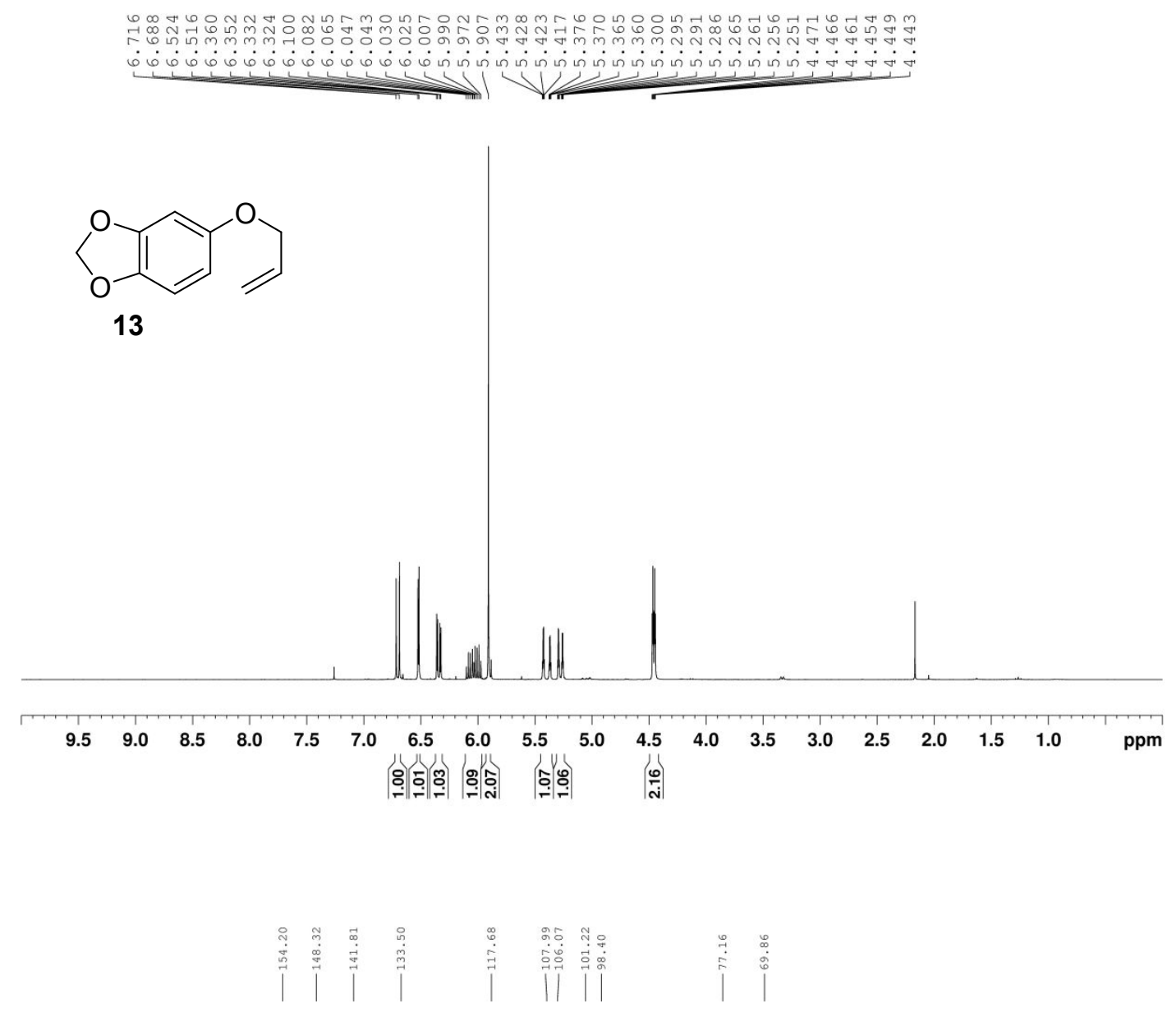<smiles>C=CCOc1ccc2c(c1)OCO2</smiles>

13

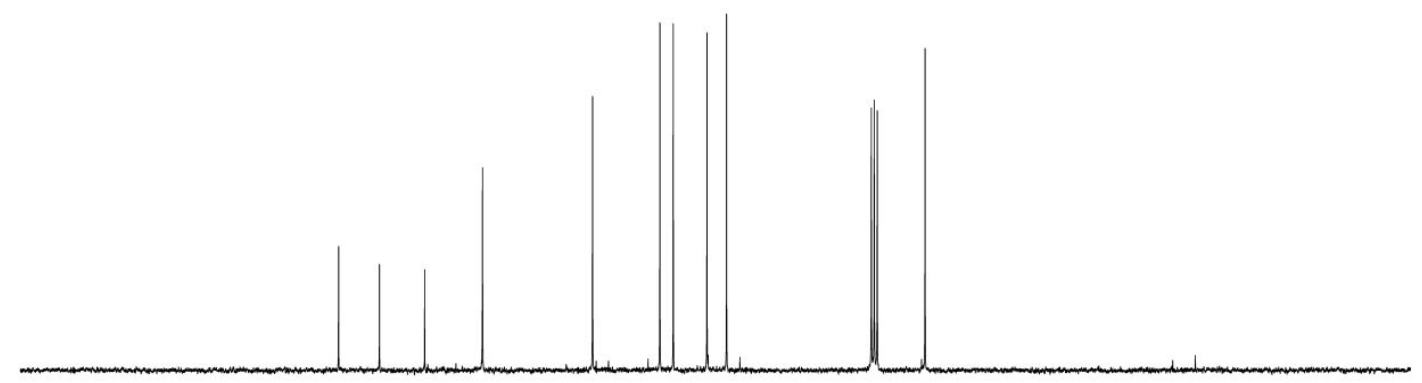

$\begin{array}{lllllllllllllllllll}190 & 180 & 170 & 160 & 150 & 140 & 130 & 120 & 110 & 100 & 90 & 80 & 70 & 60 & 50 & 40 & 30 & 20 & \mathrm{ppm}\end{array}$ 
<smiles>C=CCOc1cc(C=O)c2c(c1)OCO2</smiles>

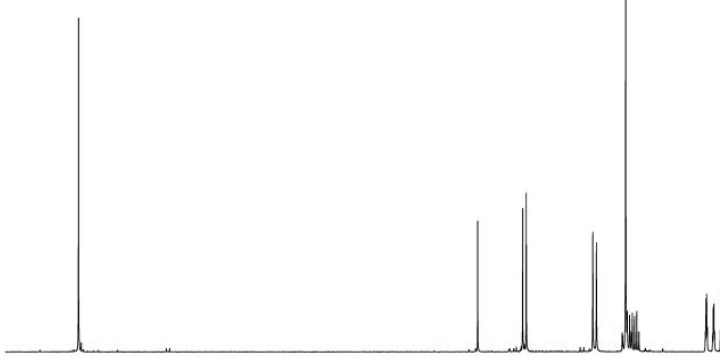

(웅
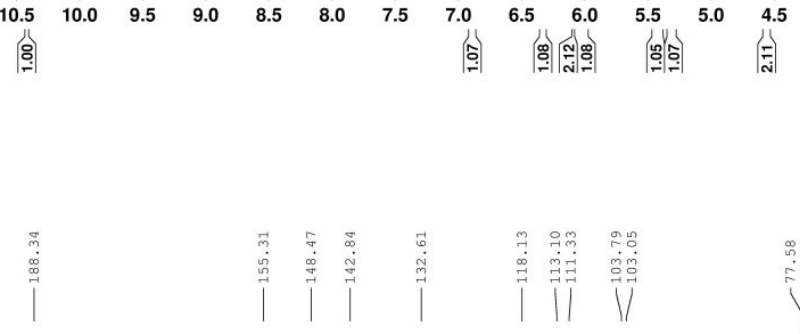<smiles>C=CCOc1cc(C=O)c2c(c1)OCO2</smiles>

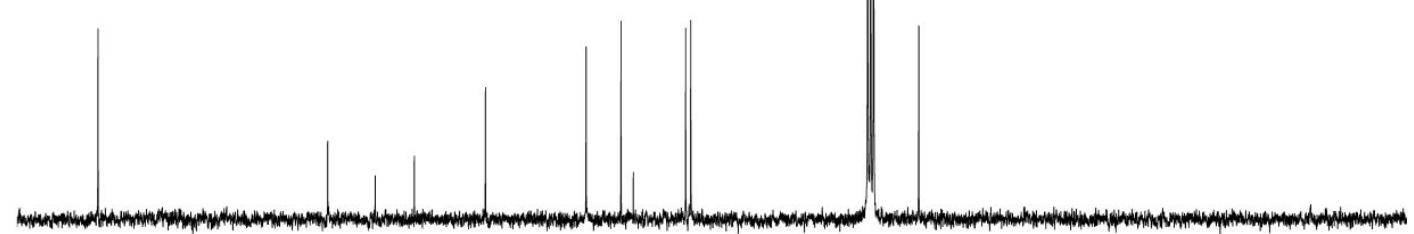

$\begin{array}{llllllllllllllllllll}190 & 180 & 170 & 160 & 150 & 140 & 130 & 120 & 110 & 100 & 90 & 80 & 70 & 60 & 50 & 40 & 30 & 20 & 10 & \mathrm{ppm}\end{array}$ 

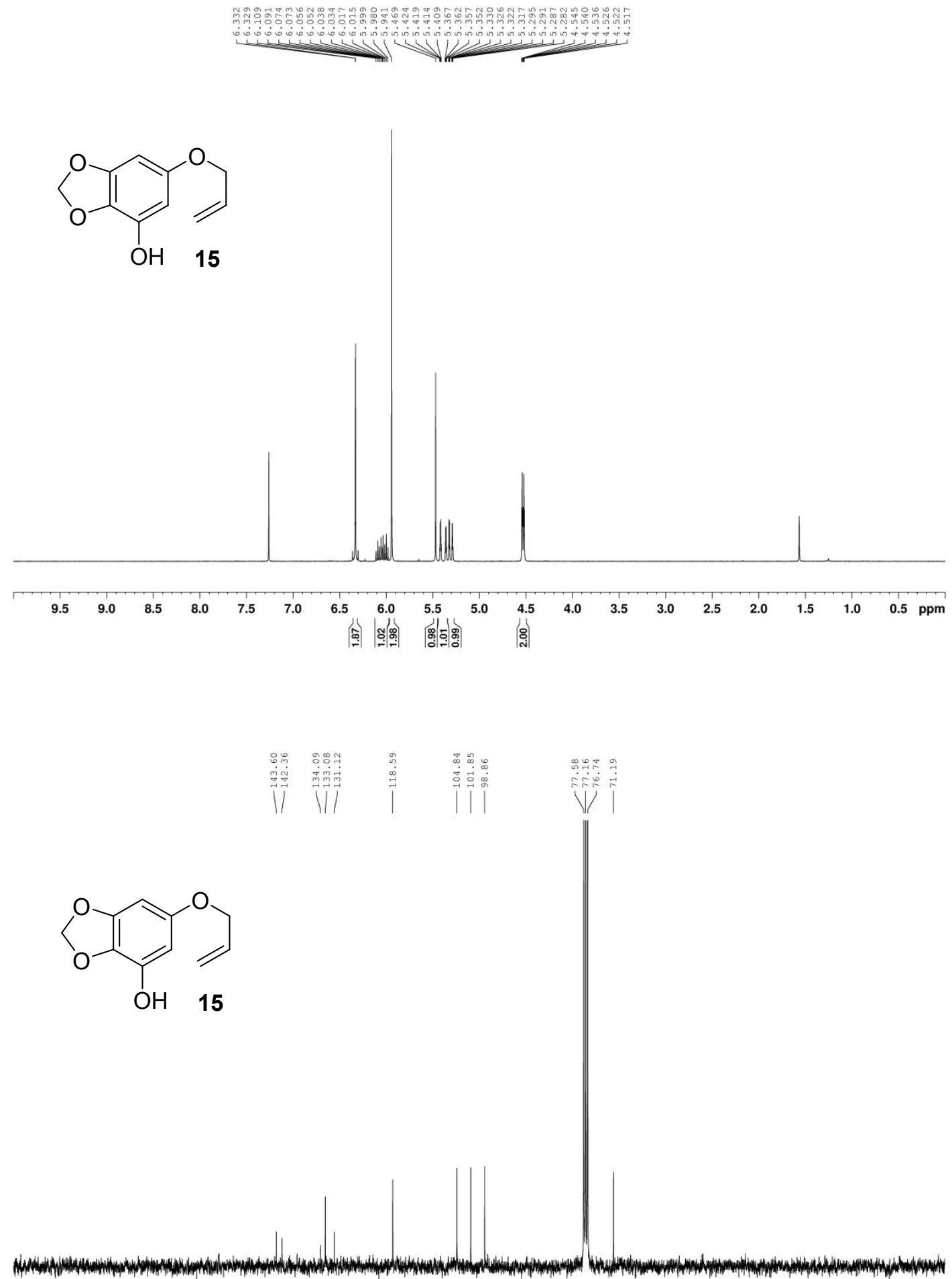

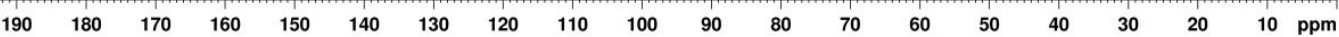



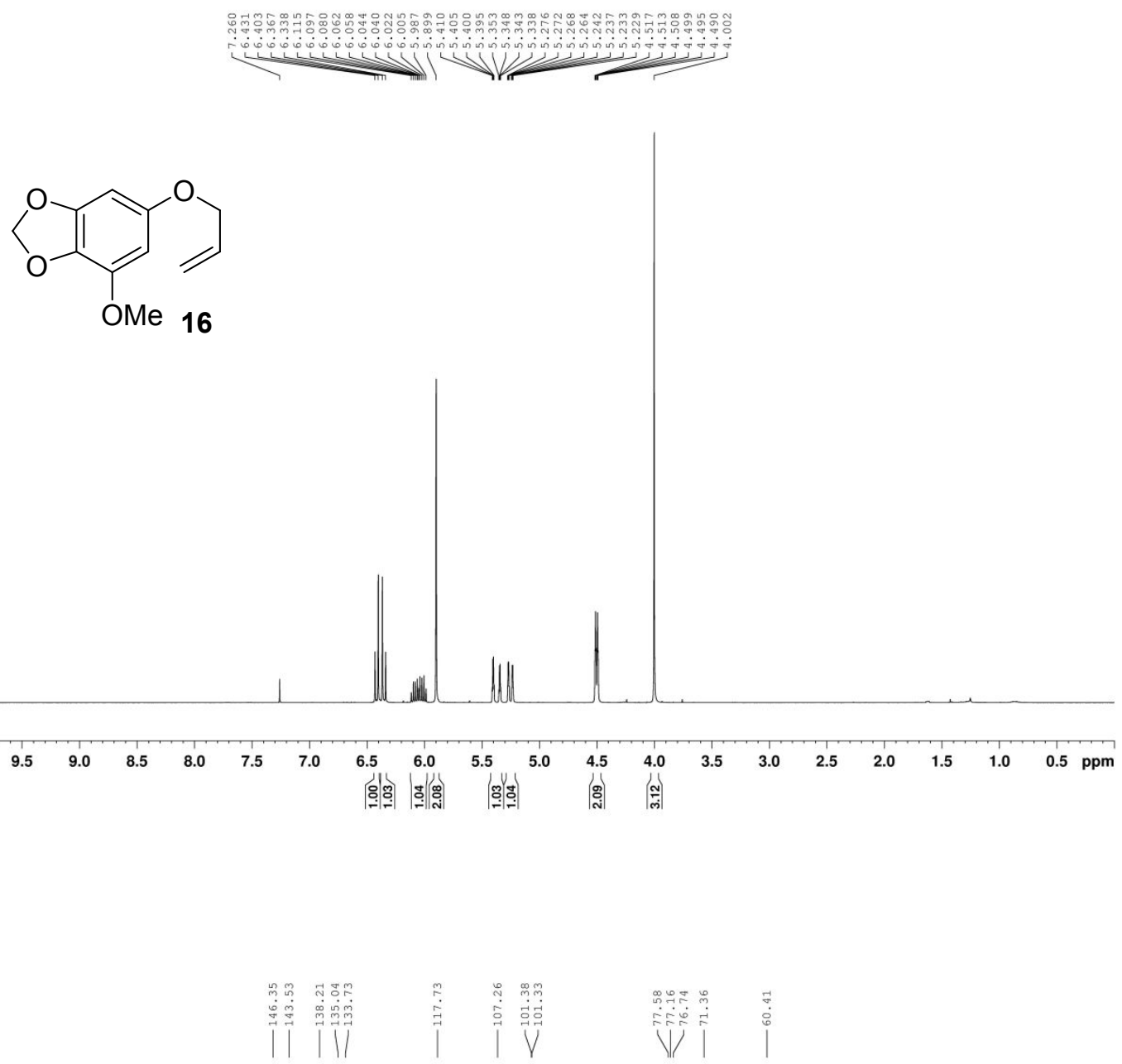<smiles>C=CCOc1cc(OC)c2c(c1)OCO2</smiles>

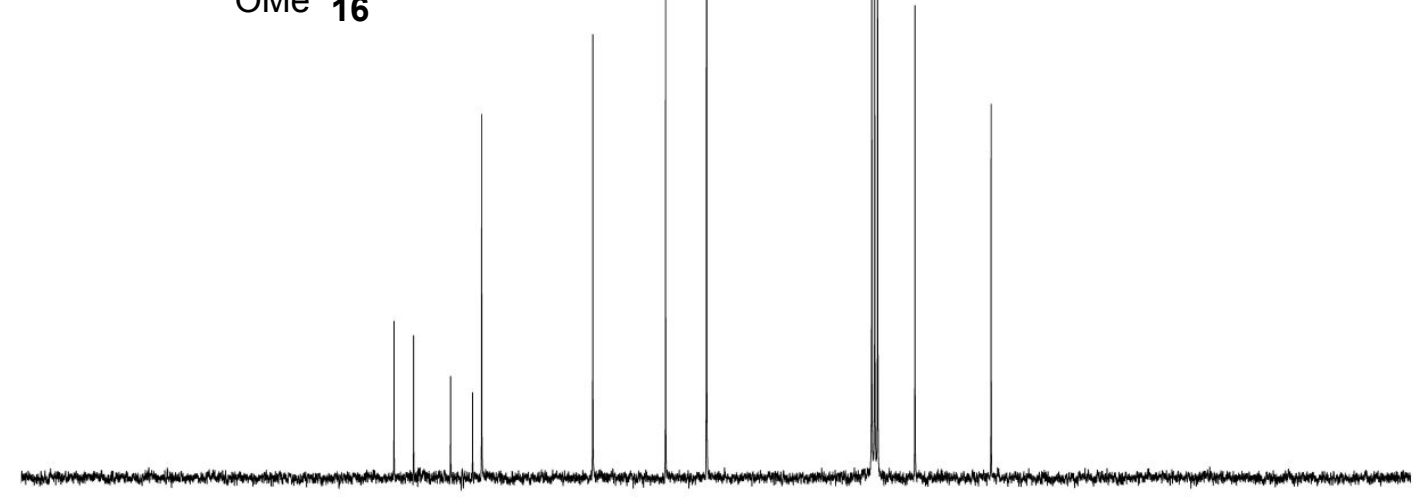

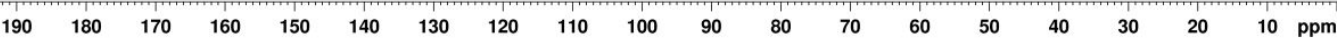



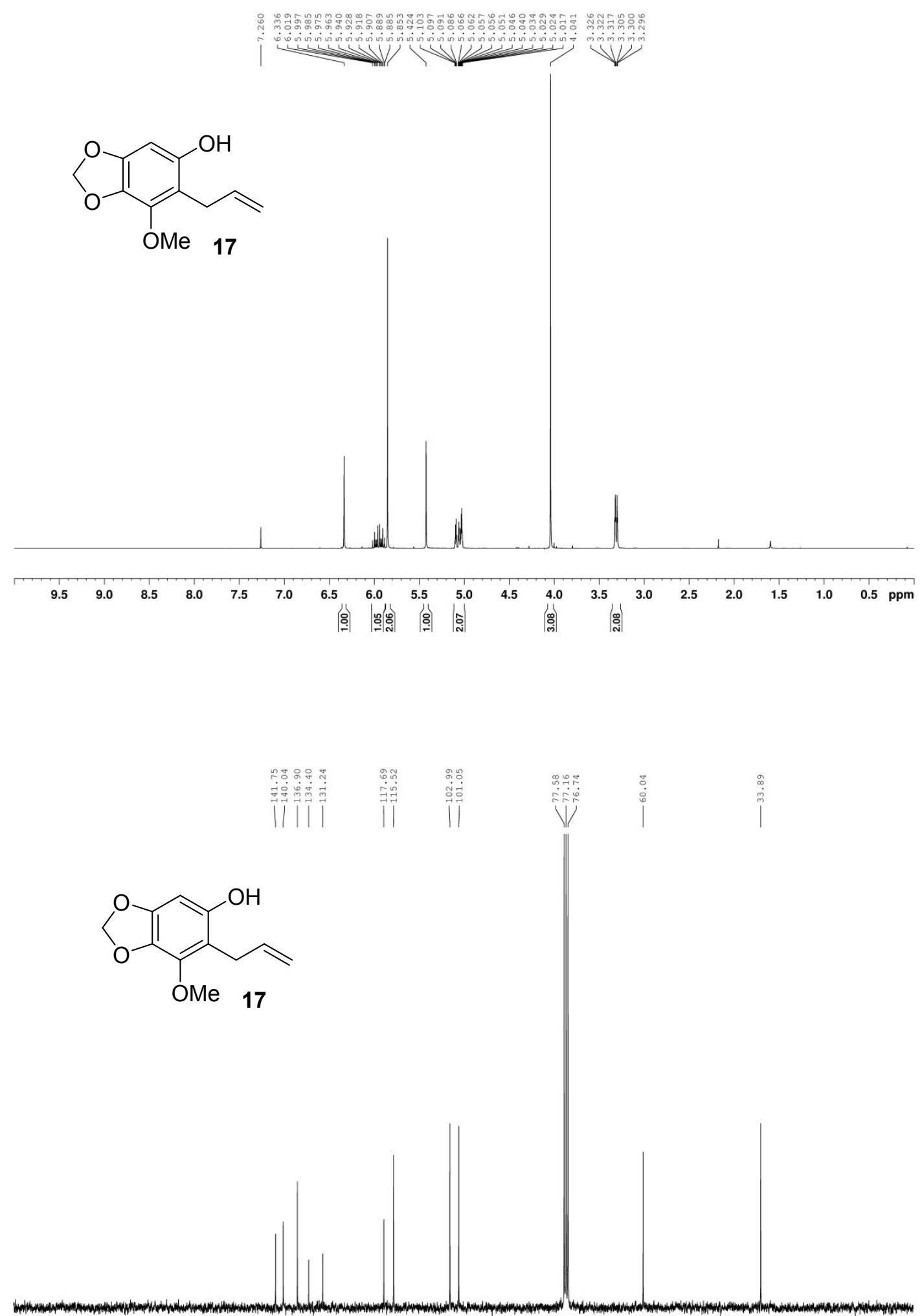

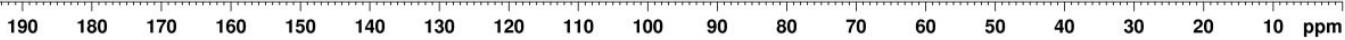



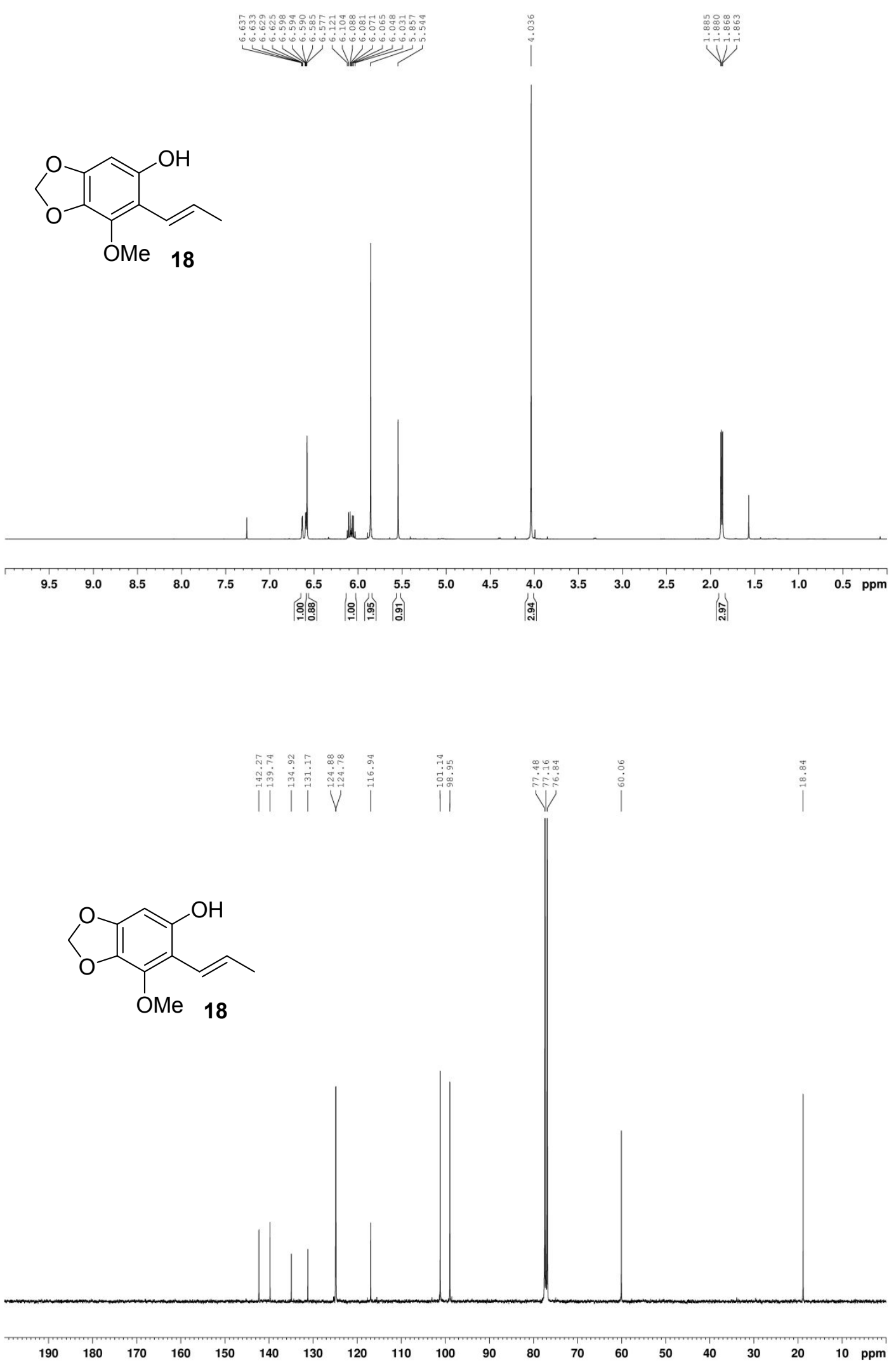


\section{viving}

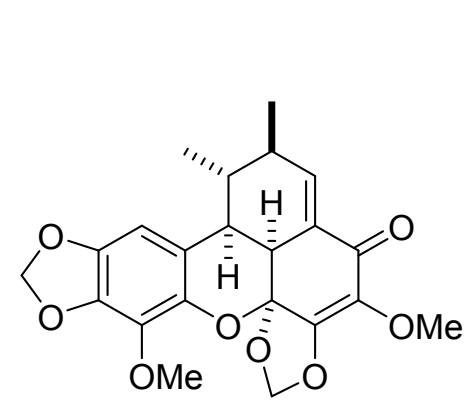

Polemannone B - 3
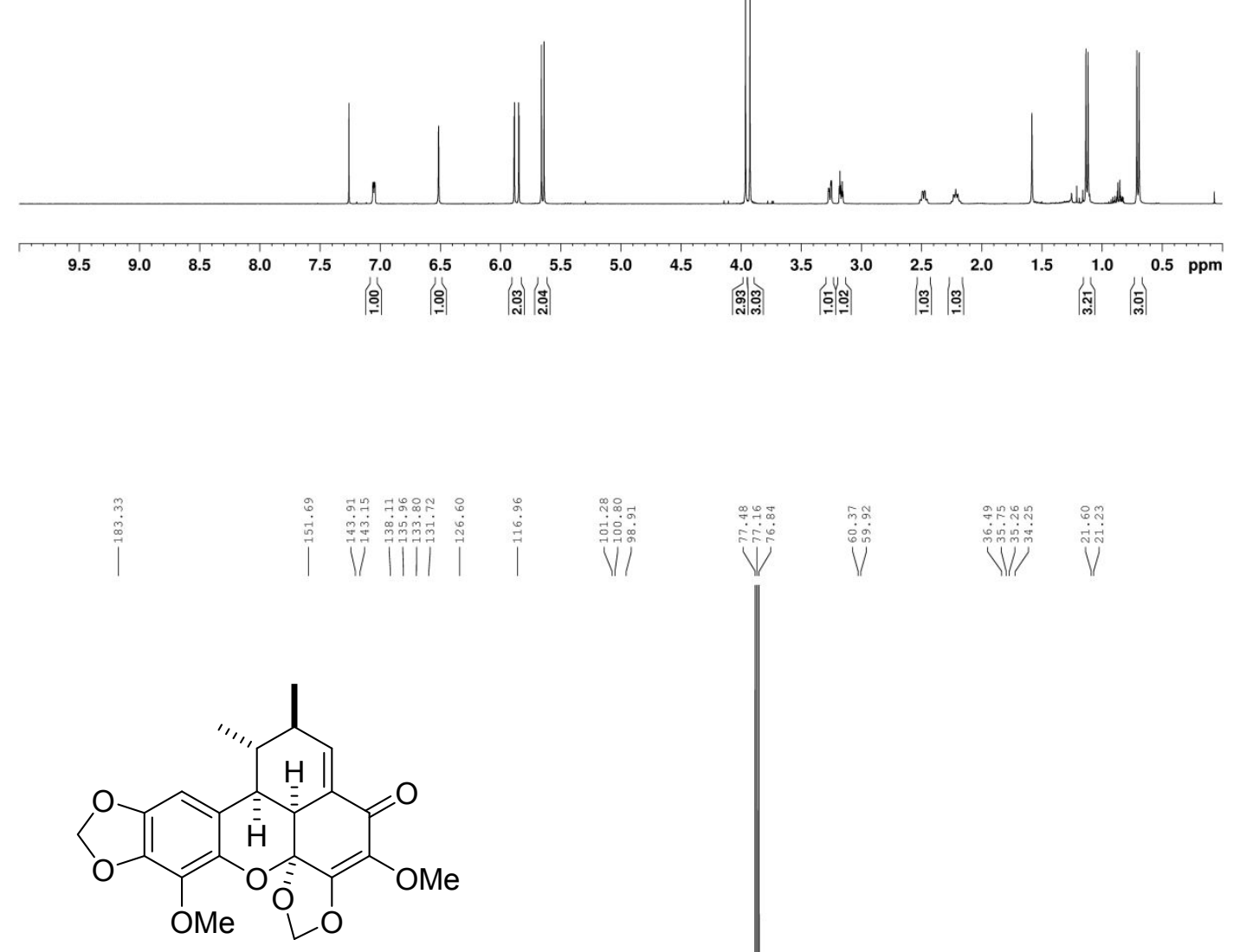

Polemannone B - 3

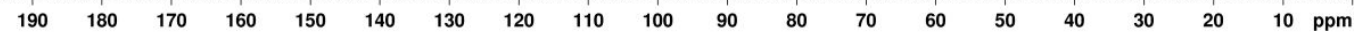




\section{Reference}

(1) Cortés-Borda, D.; Kutonova, K. V.; Jamet, C.; Trusova, M. E.; Zammattio, F.; Truchet, C.; Rodriguez-Zubiri, M.; Felpin, F.-X. Optimizing the Heck-Matsuda Reaction in Flow with a Constraint-Adapted Direct Search Algorithm. Org. Process Res. Dev. 2016, 20, 1979-1987. (2) Cortés-Borda, D.; Wimmer, E.; Gouilleux, B.; Barré, E.; Oger, N.; Goulamaly, L.; Peault, L.; Charrier, B.; Truchet, C.; Giraudeau, P.; Rodriguez-Zubiri, M.; Le Grognec, E.; Felpin, F.X. An Autonomous Self-Optimizing Flow Reactor for the Synthesis of Natural Product Carpanone. J. Org. Chem. 2018, 83, 14286-14299. 